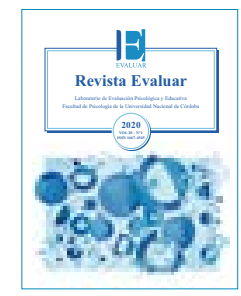

\title{
Evaluación de impulsividad por múltiples informantes: Escala de Impulsividad de Dominio Específico en Niños
}

\author{
Assessment of Impulsivity by Multiple Informants: \\ Domain-Specific Impulsivity Scale for Children
}

\author{
Eliana Vanesa Zamora ${ }^{1 *}$, Macarena del Valle ${ }^{1}$, Juan Ignacio Galli ${ }^{1}$, \\ Santiago Vernucci ${ }^{1}$, Lorena Canet-Juric ${ }^{1}$ y María Richard's ${ }^{1}$ \\ 1- Instituto de Psicología Básica, Aplicada y Tecnología (IPSIBAT - UNMDP - CONICET). Argentina.
}

Introducción

Método

Resultados

Discusión

Referencias

Recibido: 27/02/2020 Revisado: 05/05/2020 Aceptado: 09/05/2020

\section{Resumen}

El presente estudio tiene como objetivo brindar evidencias de validez acerca de la adaptación al español de la Domain Specific Impulsivity Scale for Children, la cual permite evaluar la impulsividad en el dominio interpersonal y escolar a través de diferentes informantes (niño, docentes y padres). Participaron del estudio 502 niños de 8 a 12 años. Los resultados mostraron un buen ajuste para un modelo de dos factores, escolar e interpersonal, discriminados en cada uno de los informantes. El análisis de validez convergente mostró que a mayor impulsividad reportada por los informantes en el dominio escolar, menor fue el desempeño de los niños en las pruebas de habilidades académicas. También se encontraron asociaciones con medidas de conductas problema en el aula. Contar con información sobre las propiedades de esta escala permite la evaluación de la impulsividad en el dominio interpersonal y escolar en niños argentinos.

Palabras clave: impulsividad, dominio escolar, dominio interpersonal, niños, validez

\begin{abstract}
This study aims to analyze the validity of the Spanish adaptation of the Domain Specific Impulsivity Scale for Children. This scale assesses impulsivity at the interpersonal and school domains through different informants (children, teachers, and parents). 502 children aged 8 to 12 participated in the study. The results showed a good fit for a two-factor model -school and interpersonal-, discriminated in each of the informants. The convergent validity analysis showed that, the greater the impulsivity reported by the informants in the school domain, the lower the children's performance in academic skills tests. Moreover, associations were found with measures of problem behaviors in the classroom. Having information on the psychometric properties of this scale allows the evaluation of impulsivity in the interpersonal and school domains in Argentinean children.
\end{abstract}

Keywords: impulsivity, school domain, interpersonal domain, children, validity

*Correspondencia a: Eliana V. Zamora. UNMDP- Facultad de Psicología, Deán Funes 3280 - Cuerpo V - Nivel III (7600). Tel: +54 223 4752526. Mar del Plata, Argentina. E-mail: eliana.zamora@conicet.gov.ar

Nota de autor: Este trabajo fue financiado parcialmente con fondos del Consejo Nacional de Investigaciones Científicas y Técnicas (CONICET). Conflicto de intereses: Los autores de este trabajo declaran que no existe conflicto de intereses.

Cómo citar este artículo: Zamora, E. V., del Valle, M., Galli, J. I., Vernucci, S., Canet-Juric, L., \& Richard's, M. (2020). Evaluación de la impulsividad a través de múltiples informantes: Evidencias de validez de la Escala de Impulsividad de Dominio Específico en Niños. Revista Evaluar, 20(2), 35-50. 


\section{Introducción}

La impulsividad ha sido definida como una acción no reflexiva impulsada por un estímulo, que puede resultar adaptativa o desadaptativa según el contexto y su grado de inflexibilidad. A su vez, se la ha definido como la tendencia a optar por una recompensa inmediata en detrimento de una meta u objetivo valioso a largo plazo (Nigg, 2017). Según Tsukayama, Duckworth y Kim (2013), la impulsividad también se define, de manera general, como la incapacidad de regular el comportamiento, la atención e incluso las emociones al servicio de metas valoradas. Los autores señalan que la impulsividad y su anverso, el autocontrol, pueden considerarse como dos extremos del mismo continuo (Duckworth \& Kern, 2011). Así, el autocontrol se define como la capacidad de anular o modificar los impulsos de manera voluntaria y supone aplicar un esfuerzo para regular el comportamiento, el pensamiento y las emociones a fin de alcanzar metas a largo plazo (Baumeister, Vohs, \& Tice, 2007; De Ridder, Lensvelt-Mulders, Finkenauer, Stok, \& Baumeister, 2012).

El concepto de impulsividad es ampliamente utilizado en la literatura. Se encuentra presente en los principales modelos de personalidad, en las aproximaciones explicativas del comportamiento humano (Squillace \& Picón-Janeiro, 2017) e, incluso, representa uno de los criterios diagnósticos más comúnmente empleados en el Manual Diagnóstico y Estadístico de los Trastornos Mentales (5 a ed. [DSM-5]; Few, Lynam, \& Miller, 2015). Las concepciones acerca de la impulsividad varían significativamente de acuerdo con diferentes enfoques teóricos (ver Squillace, Picón-Janeiro, \& Schmidt, 2011). Se han propuesto al menos una docena de modelos de impulsividad (ver Duckworth \& Kern, 2011), entre los que se destacan el modelo de Eysenck y Eysenck (1977), el modelo de Zuckerman (2007), el modelo biopsicoso- cial de Barrat, Stanford, Kent y Felthous (1997), el modelo multifactorial de Whiteside y Lynam (2001) y el modelo específico de dominio de Tsukayama et al. (2013). Así como existen varios modelos teóricos sobre la impulsividad, se han desarrollado varios instrumentos basados en ellos: el Cuestionario de Impulsividad de Eysenck (Eysenck \& Eysenck, 1977), la Escala de Búsqueda de Sensaciones de Zuckerman (Zuckerman, Eysenck, \& Eysenck, 1978), la Escala de Impulsividad de Barratt (Patton, Stanford, \& Barratt, 1995) y, por último, la Escala de Comportamiento Impulsivo UPPS (Whiteside \& Lynam, 2001). Con respecto al estudio de esta cuestión en población infantil, Zapolski, Stairs, Settles, Combs y Smith (2010) realizaron un estudio con niños de 7 a 13 años sobre cuatro de los cinco rasgos de modelo UPPS de Whiteside y Lynam (2001). El trabajo mostró que las distintas subescalas del modelo se asociaban con conductas de riesgo, agresión, estado de ánimo negativo, problemas atencionales y con dificultades académicas ( $r$ entre .28 y .52).

Por otro lado, diferentes autores señalan que los comportamientos impulsivos pueden presentar variaciones de acuerdo con los contextos en los que se ve implicado un niño. Tsukayama et al. (2013) señalan que un niño puede ser impulsivo en un dominio en particular (p. ej., el hogar) porque allí se le presentan demandas específicas en cuanto a la gratificación de sus impulsos, de modo tal que sus recursos cognitivos y/o motivacionales no resultan suficientes para contrarrestarlos. Sin embargo, en otro dominio (p. ej., la escuela) puede no ocurrirle lo mismo y su capacidad para controlar los impulsos puede funcionar de manera más adaptativa (Wilkowski \& Robinson, 2016). Inclusive, Mischel, Shoda y Mendoza-Denton (2002) indican que pueden construirse distintos perfiles; por ejemplo, un niño puede cumplir con las tareas académicas en el aula pero, en situaciones interpersonales, tener conductas 
más impulsivas. De este modo, señalan que los dominios o contextos situacionales no son lo mismo que los procesos psicológicos. La cuestión central para estos autores radica en determinar si tiene sentido referirse a la impulsividad como un constructo de dominio general o si, en cambio, los comportamientos impulsivos están tan estrechamente vinculados a factores situacionales que sería conveniente referirse a la impulsividad como un constructo de dominio específico.

Uno de los primeros estudios que indagó esta cuestión fue el realizado por Humphrey (1982), en el que docentes de niños de 9 y 10 años de edad fueron entrevistados acerca de diferentes comportamientos impulsivos en niños. Los análisis factoriales revelaron que los comportamientos podían agruparse en dos factores principales: comportamientos orientados al dominio cognitivo (a nivel personal) y comportamientos orientados hacia otros (a nivel interpersonal). En el primer dominio se consideraron acciones impulsivas tales como no completa las tareas cuando el adulto no está mirando o se distrae de tareas o responsabilidades; por otro lado, cuestiones como entra en discusiones y/o peleas con otros niños o habla fuera de turno fueron ejemplos del dominio interpersonal.

Bajo el supuesto de que el comportamiento impulsivo en el contexto social e interpersonal está relacionado con el comportamiento en el contexto escolar (aunque es distinto de aquel), Tsukayama et al. (2013) desarrollaron la Domain Specific Impulsivity Scale for Children (DSIS-C), la cual, además de brindar un puntaje general sobre impulsividad, proporciona información específica en el dominio interpersonal y escolar. Para el desarrollo de esta escala, se tomaron en consideración conductas específicas descritas por los niños. Asimismo, esta medida cuenta con tres formas paralelas de evaluación, correspondientes a cada informante: los docentes, los padres y los propios niños. La escala DSIS-C mostró un ajuste adecuado según el modelo teórico propuesto tanto en las respuestas de los niños como en las de los docentes y de los padres (niño, $\chi_{(19)}^{2}=141.36$, $p<.001 ; \mathrm{CFI}=.90, \mathrm{RMSEA}=.09$; docente, $\chi_{(19)}^{2}$ $=362.94, p<.001 ; \mathrm{CFI}=.92$, RMSEA = .16; padres, $\chi_{(19)}^{2}=85.37, p<.001$; CFI $=.95$, RM$\mathrm{SEA}=.08)$. Es decir que, independientemente del informante, se encontraron dos factores discriminados: impulsividad interpersonal e impulsividad escolar. Los índices de confiabilidad de la escala fueron entre adecuados y muy buenos ( $\alpha$ entre .77 y .95) para docentes y padres; la medida de los niños presentó los menores niveles de confiabilidad ( $\alpha$ entre .63 y .73). Este estudio también indicó que los dominios escolar e interpersonal de la DSIS-C variaban en sus asociaciones con otras conductas. Así, para cada uno de los informantes, la impulsividad en el dominio escolar se relacionó de manera inversa con la autoconciencia y directa con la cantidad de horas de estudio y el desempeño académico, mientras que, en el dominio interpersonal, se relacionó de manera directa con la agresión e inversamente con la agradabilidad (Tsukayama et al., 2013).

En el ámbito escolar, los niños suelen experimentar conflictos entre objetivos académicos que valoran a largo plazo y objetivos no académicos que son más gratificantes en el momento, lo que lleva a un esfuerzo para realizar y concretar sus actividades escolares (Duckworth, Taxer, Eskreis-Winkler, Galla, \& Gross, 2019). En este sentido, Duckworth, Kim y Tsukayama (2013) hallaron que los niños consideran que realizar las tareas del hogar o estudiar para un examen son actividades sustancialmente menos agradables que otras actividades cotidianas, pero, al mismo tiempo, consideran que cumplir con las demandas académicas es más importante para sus objetivos a largo plazo que cualquier otra actividad. También, en este estudio, se encontró que este patrón es similar independientemente del género, del nivel 
educativo, del tipo gestión de las escuelas (públicas o privadas) e incluso de las calificaciones escolares. Respecto del tipo de informante, aunque en ocasiones esto puede resultar problemático (por ejemplo, los padres pueden expresar características propias en lugar de la personalidad de su hijo o brindar respuestas basadas en preconceptos sobre el desarrollo típico de la infancia; Guion, Mrug, \& Windle, 2009), ciertos autores (p. ej., Klonsky, Jane, Turkheimer, \& Oltmanns, 2002) coinciden en señalar que los docentes y los padres representan fuentes primarias de información en entornos aplicados (como las escuelas y el hogar). Además, tener varios informantes brinda una perspectiva más amplia y comprehensiva, ya que permite indagar acerca de las conductas de los niños en los diferentes contextos en los que pueden manifestarse (De los Reyes, Thomas, Goodman, \& Kundey, 2013). De manera similar, esta postura es consistente con una extensa investigación que demuestra la validez de dos dominios diferentes en los cuales se manifiesta uno de los trastornos con mayor prevalencia en la población infantil: el Trastorno de Déficit de Atención con Hiperactividad (TDAH). Willcutt et al. (2012) encontraron que puede distinguirse un contexto o dominio interpersonal donde se manifiesta la sintomatología de manera desadaptativa (p. ej, a menudo interrumpe a otros o se entromete) y un dominio o contexto escolar donde también estas conductas irrumpen en el aula (p. ej, a menudo se mueve nerviosamente o da golpecitos con las manos o los pies, o se retuerce en el asiento), lo que muestra que la sintomatología necesaria para el diagnóstico de TDAH debe presentarse, al menos, en estos dos dominios de la vida cotidiana del niño.

La escala DSIS-C fue traducida al español como la Escala de Impulsividad Específica de Dominio para Niños (EIEDN) y adaptada con una muestra de niños argentinos de 8 a 12 años. Los datos obtenidos en este estudio indicaron una consistencia interna adecuada para los tres informantes (niños: $\alpha=.64$; docentes: $\alpha=.86$; padres: $\alpha=$.76; Richard's et al., 2017). Recientemente, Canet-Juric, Galli, Zaidán y Vázquez-Echeverría (2019) utilizaron la adaptación de la escala DSIS-C para analizar su relación con calificaciones escolares de Prácticas del lenguaje y Matemática, y con tareas estandarizadas de comprensión lectora y cálculo aritmético en niños. Sin embargo, el énfasis de estos trabajos no estuvo orientado hacia el análisis de la estructura teórico-conceptual del modelo de la escala DSIS-C.

A pesar de la importancia que la impulsividad tiene en el ámbito académico y en la vida cotidiana de los niños, la literatura se ha centrado en explicar las diferencias individuales en la conducta a partir de aspectos tales como los rasgos de personalidad o el temperamento. De este modo, contar con una versión adaptada, válida y confiable de la DSIS-C para ser aplicada a población infantil argentina, contribuiría a la comprensión de la conducta de los niños en edad escolar, lo cual reviste un gran valor clínico, diagnóstico, e incluso, educativo. Además, el análisis de la estructura de la escala supondría un aporte empírico a la indagación del modelo teórico del instrumento. Por este motivo, este trabajo se propone presentar evidencias de validez de la EIEDN, que consiste en la traducción y adaptación al español de la DSIS-C de Tsukayama et al. (2013). Para efectuar los análisis, se consideraron los criterios propuestos por Sireci (2003), quien sugiere que para establecer la validez de un instrumento, debe considerarse cuál es el principal propósito del mismo. En este caso, el propósito de la EIEDN es evaluar la impulsividad (general, interpersonal y escolar) a través de diferentes informantes en población infantil.

Para analizar la validez del instrumento se plantearon tres objetivos: (1) analizar la estructura factorial de la escala mediante análisis confirmatorio (validez de constructo) y verificar su 
confiabilidad, (2) analizar la validez externa (concurrente) del instrumento mediante su asociación con otras medidas que evalúan el mismo constructo (transgresión de las normas, impulsividad, hiperactividad y déficit atencional) y (3) analizar la validez convergente de la escala de impulsividad en el dominio escolar mediante su asociación con variables teóricamente relacionadas (desempeño en tareas estandarizadas de comprensión lectora y cálculo aritmético).

\section{Metodología \\ Diseño y participantes}

Se utilizó un diseño correlacional, no experimental y transversal (Hernández-Sampieri, Fernández-Collado, \& Baptista-Lucio, 2014). La muestra fue no probabilística intencional. Se llevaron a cabo tres estudios en función de los objetivos propuestos. La muestra general y utilizada para analizar la validez de constructo del instrumento (Objetivo 1) estuvo compuesta por 502 niños en edad escolar, de entre 8 y 12 años $(\mathrm{M}=10.78, \mathrm{DE}=1.56)$. Del total, 238 eran niños $(47.41 \%)$ y 264 eran niñas (52.59\%). Además, 114 niños se encontraban en el $3^{\circ}$ grado $(22.71 \%)$, 139 en el $4^{\circ}$ grado $(27.69 \%), 150$ en el $5^{\circ}$ grado (29.88\%) y 99 en el $6^{\circ}$ grado (19.72\%) de escolaridad primaria básica. También se obtuvieron las respuestas de 354 padres acerca de la conducta de esos niños (podría responder el padre, la madre, o el tutor del niño, pero solo hubo un informante por cada niño). Finalmente, se obtuvieron 197 respuestas de siete docentes sobre sus alumnos: una maestra de $3^{\circ}$ grado completó 35 respuestas (17.8\%), dos maestras de $4^{\circ}$ grado completaron 74 respuestas $(37.6 \%)$, dos maestras de $5^{\circ}$ grado completaron 40 respuestas $(20.3 \%)$ y dos maestras de $6^{\circ}$ grado completaron 48 respuestas $(24.4 \%)$.

Para el logro de los Objetivos 2 y 3 , se uti- lizaron dos submuestras. Para analizar la validez concurrente del instrumento (Objetivo 2), la segunda muestra estuvo compuesta enteramente por docentes. Se contó con la participación de 3 docentes que respondieron sobre 57 niños de $3^{\circ}$ grado $(\mathrm{n}=20), 4^{\circ}$ grado $(\mathrm{n}=24)$ y $5^{\circ}$ grado $(\mathrm{n}$ $=12$ ). Por último, la tercera muestra, utilizada para analizar la validez convergente de la escala (Objetivo 3), estuvo compuesta por 76 respuestas de niños (28 niños y 48 niñas; $4^{\circ}$ grado: $\mathrm{n}=$ 23 ; $5^{\circ}$ grado: $\mathrm{n}=30 ; 6^{\circ}$ grado: $\mathrm{n}=23$ ), 63 respuestas de sus padres (padre, madre o tutor) y 73 respuestas de 3 docentes sobre esos niños ( $4^{\circ}$ grado: $\mathrm{n}=23 ; 5^{\circ}$ grado: $\mathrm{n}=28 ; 6^{\circ}$ grado: $\mathrm{n}=22$ ).

Todos los niños participantes asistían a escuelas de gestión privada de zonas residenciales de la ciudad de Mar del Plata, Argentina. Se consideraron los siguientes criterios de inclusión de participantes: estudiantes que no están bajo tratamiento psicológico y/o psiquiátrico, con visión normal o corregida, y que presentan un desarrollo típico, sin déficits ni alteraciones, y sin antecedentes de trastornos del aprendizaje o del desarrollo.

\section{Instrumentos}

Escala de impulsividad. Se utilizó la Escala de Impulsividad para Niños Específica de Dominio (EIEDN; Richard's et al., 2017) que consiste en la traducción y adaptación al español de la DSIS-C de Tsukayama et al. (2013). El instrumento permite indagar el comportamiento impulsivo en diferentes contextos o dominios como el social y el escolar. Los protocolos para padres, docentes y niños constan de 8 ítems cada uno, que representan comportamientos frecuentes en los niños. Cada ítem cuenta con cinco opciones de respuestas posibles que indagan la frecuencia con la que ha realizado una conducta: casi nunca, aproximadamente una vez por mes, aproximadamente dos 
o tres veces al mes, aproximadamente una vez por semana y casi todos los días.

Conductas en el aula. Para la evaluación de conductas problema en el aula se utilizaron las subescalas transgresión, impulsividad, hiperactividad y déficit atencional de la Guía de Observación Comportamental (GOC; Ison \& Fachinelli, 1993). La GOC es completada exclusivamente por el docente del niño y permite obtener datos acerca de la frecuencia de aparición de una serie de conductas problema, con tres opciones de respuesta: nunca, a veces y casi siempre. El factor transgresión implica la presencia de un patrón persistente de conductas en el que se violan los derechos básicos de los demás y las normas sociales apropiadas a la edad. Este factor está compuesto por nueve ítems (por ejemplo: grita en el aula/casa al solicitar alguna cosa de los demás, incita a la agresión, se burla de otros). El factor impulsividad involucra la pérdida de la capacidad para inhibir la conducta frente a un estímulo cuando es necesario hacerlo. Está conformado por cinco ítems (por ejemplo: adopta decisiones repentinas sin pensar en las consecuencias, da respuestas precipitadas antes de que se le acaben de formular las preguntas). El factor hiperactividad implica conductas motoras y/o verbales de tasa elevada que aparecen sin un aparente control de estímulos y que, por mantenerse durante un largo período, difícilmente pueden ser ignoradas por otras personas. Está compuesto por cuatro ítems (por ejemplo: cambia de una actividad a otra dejando ambas incompletas, le cuesta respetar el turno en juegos o actividades grupales). Por último, el factor déficit atencional se refiere a la dificultad por parte del niño para mantener focalizada su atención durante períodos cortos. Este factor está compuesto por cuatro ítems (por ejemplo: pierde cosas necesarias para una tarea o actividad escolar, dificultad para concentrarse en el trabajo escolar). La
GOC cuenta con datos normativos obtenidos en nuestro país (Ison \& Soria, 1997), posee evidencias sobre su validez discriminante (diferenciando grupos con y sin conductas problema; Ison \& Fachinelli, 1993) y ha sido administrada en población infantil en diversos estudios (p. ej., Andrés, 2014; Morelato \& Ison, 2002; Musso, 2009).

Cálculo Aritmético. Para evaluar la habilidad de cálculo se utilizó el subtest de aritmética de la Prueba de Logro de Amplio Rango WRAT-3 de Wilkinson (1993). Este subtest evalúa las habilidades básicas de conteo, lectura de símbolos numéricos y desempeño en cálculos escritos. Está compuesto por 55 ítems que representan cálculos aritméticos cuya dificultad aumenta gradualmente, desde problemas de adición de un dígito hasta problemas avanzados de álgebra. El WRAT-3 ha mostrado índices de validez y confiabilidad adecuados en distintos grupos de edad (Snelbaker, Wilkinson, Robertson, \& Glutting, 2001), especialmente en niños de 9 a 11 años en el contexto argentino (p. ej, Vernucci, Canet-Juric, Andrés, \& Burín, 2017).

Comprensión lectora. Se utilizaron las pruebas de screening del Test Leer para Comprender (TLC; Abusamra, Ferreres, Raiter, De Beni, \& Cornoldi, 2010), el cual cuenta con dos pruebas, una para niños de 9 y 10 años (cuarto y quinto año escolar) y otra prueba de mayor dificultad para niños de 11 y 12 años de edad (sexto año escolar). Cada prueba contiene un texto narrativo y un texto expositivo sobre los que luego se presentan diez preguntas, cada una con cuatro alternativas de respuesta, entre las cuales el alumno debe marcar la correcta. Se asigna 1 punto por respuesta correcta y 0 por incorrecta. La puntuación máxima posible para cada texto es 10 . Se han reportado niveles de confiabilidad y validez adecuados (discriminante) para esta prueba en ambos grupos de edad (Abusamra et al., 2010). 
Procedimiento para la recolección de los datos y consideraciones éticas

El presente estudio fue evaluado y aprobado por el Comité de Ética del Programa Temático Interdisciplinario en Bioética, dependiente de la Secretaría de Ciencia y Técnica del Rectorado de la Universidad Nacional de Mar del Plata (Fecha 11/03/2015). Luego fue presentado y aprobado por las instituciones educativas, donde se llevaron a cabo reuniones informativas con el personal docente y con los padres/tutores de los niños sobre los objetivos y procedimientos del estudio. Se entregó una hoja de información y se invitó a los padres/tutores a participar del estudio, para lo cual debían firmar un consentimiento informado. Asimismo, los niños debían manifestar su acuerdo para participar, y se les informó que podrían abandonar el estudio en cualquier momento si así lo requerían. La evaluación fue llevada a cabo de manera individual en un aula de las instituciones educativas a la que asistían los niños y fueron realizadas por un profesional entrenado para tal fin.

En el desarrollo del presente estudio se respetaron los lineamientos dados por el Consejo Nacional de Investigaciones Científicas y Técnicas de Argentina (CONICET) para el comportamiento ético en las Ciencias Sociales y Humanidades (Resolución $N^{\circ}$ 2857, 2006), los criterios recomendados por la American Psychological Association para las actividades destinadas a obtener conocimientos sobre procesos psicológicos en seres humanos (APA, 2010) y los principios éticos para la investigación con seres humanos estipulados por la Declaración de Helsinki (World Medical Association, 2013).

\section{Análisis de los datos}

Se utilizó el Paquete Estadístico para Ciencias Sociales (SPSS; IBM, 2013) v. 22. En primer lugar, se analizó la proporción de datos faltantes en cada una de las variables. En todas, la proporción fue menor al 3\%. Luego, se utilizó el método de expectación-maximación para reemplazar los valores faltantes. Respecto de la presencia de casos atípicos, no se detectaron casos con desviaciones mayores a 3.29 DE respecto de la media en alguna o más de las variables (George \& Mallery, 2003; Tabachnick \& Fidell, 2001).

Para evaluar la validez de constructo $(\mathrm{Ob}-$ jetivo 1) se realizaron tres análisis factoriales confirmatorios (AFC) de la EIEDN, uno por cada informante. Se utilizaron para tal fin los protocolos de los 502 niños para el primer modelo, 354 protocolos de los padres para el segundo modelo, mientras que para el tercer modelo se utilizaron 197 protocolos de los docentes de los niños. Estos análisis se realizaron con el programa Lisrel (Scientific Software International, 2006). El método de estimación utilizado fue el de máxima verosimilitud (ML), y para evaluar el ajuste de los modelos, se utilizó el estadístico chi-cuadrado $\left(\chi^{2}\right)$, y los índices de ajuste CFI, NFI, NNFI y IFI, así como el RMSEA como medida de error (Bentler, 1990; Bentler \& Bonett, 1980; Hu \& Bentler, 1998). Los valores superiores a .90 para el CFI, NFI, NNFI, e IFI indican un ajuste aceptable a excelente; para el caso del RMSEA son adecuados valores inferiores a .08. Se estimó la confiabilidad compuesta ( $\rho$; Hair, Anderson, Tatham, \& Black, 1995). Los valores iguales o superiores a $\rho=.70$ son considerados como aceptables (Nunnally, 1978). Asimismo, se siguieron las sugerencias sobre el tamaño muestral necesario para la validación de instrumentos, que estiman que debe contarse con 5-10 participantes por ítem (Carretero-Dios \& Pérez, 2005). Además, se analizaron las correlaciones de los dominios entre los informantes a través del coeficiente de correlación $r$ de Pearson.

En segundo lugar, para evaluar la validez 
concurrente (Objetivo 2) se realizaron correlaciones $r$ de Pearson entre la versión docente de la EIEDN y las subescalas transgresión a las normas, impulsividad, hiperactividad y déficit atencional de la GOC. En último lugar, y con el fin de analizar la validez convergente (Objetivo 3), se realizaron correlaciones de $r$ de Pearson entre impulsividad escolar (medida a través de padres y docentes) y el desempeño de los niños en cálculo matemático y comprensión lectora. Debido a la baja fiabilidad del cuestionario de impulsividad respondido por los niños, solo se analizó lo informado por el docente y por los padres.

\section{Resultados}

\section{Objetivo 1: Validez de constructo}

Se realizaron tres AFC para probar el modelo bifactorial en cada uno de los informantes:
(1) respuestas de los niños, (2) respuestas de los padres acerca de sus hijos, (3) respuestas de los docentes acerca de sus alumnos. La Tabla 1 muestra el resultado de ajuste para los tres modelos, conjuntamente con las cargas factoriales y la confiabilidad de cada subescala para cada informante.

Se puede observar que la escala mostró un ajuste excelente del modelo de dos factores para cada uno de los informantes. El Factor 1, compuesto por 4 ítems, se refiere a la impulsividad en el dominio escolar y obtuvo un índice de confiabilidad baja para el niño $(\rho=.53)$, y adecuados para el docente $(\rho=.95)$ y el padre $(\rho=.80)$, mientras que el Factor 2 estuvo compuesto por 4 ítems referidos a la impulsividad interpersonal y obtuvo valores adecuados de confiabilidad para el niño $(\rho=.69)$, el docente $(\rho=.92)$ y el padre $(\rho=.77)$. Como se observa en la Tabla 1 , los indicadores de ajuste del modelo fueron satisfactorios en los tres

Tabla 1

Escala de Impulsividad Específica de Dominio para Niños (EIEDN): ítems y cargas estandarizadas para cada informante.

\begin{tabular}{|c|c|c|c|c|c|c|}
\hline & \multicolumn{2}{|c|}{ Niño } & \multicolumn{2}{|c|}{ Docente } & \multicolumn{2}{|c|}{ Padre } \\
\hline & $\begin{array}{c}\text { Factor } \\
1\end{array}$ & $\begin{array}{l}\text { Factor } \\
2\end{array}$ & $\begin{array}{c}\text { Factor } \\
1\end{array}$ & $\begin{array}{c}\text { Factor } \\
2 \\
\end{array}$ & $\begin{array}{c}\text { Factor } \\
1 \\
\end{array}$ & $\begin{array}{c}\text { Factor } \\
2\end{array}$ \\
\hline Ítem 1. Me olvido cosas o elementos que necesito para clases. & .47 & & .94 & & .66 & \\
\hline $\begin{array}{l}\text { Ítem } 4 \text {. No puedo encontrar cosas porque mi banco o mi habitación } \\
\text { están muy desordenados. }\end{array}$ & .38 & & .86 & & .51 & \\
\hline Ítem 6. No recuerdo las cosas que mi maestra me pide hacer. & .49 & & .98 & & .80 & \\
\hline $\begin{array}{l}\text { Ítem } 7 \text {. En vez de escuchar o prestar atención me pongo a pensar en } \\
\text { otras cosas. }\end{array}$ & .54 & & .87 & & .82 & \\
\hline Ítem 2. Interrumpo a mis compañeros mientras están hablando. & & .56 & & .80 & & .56 \\
\hline $\begin{array}{l}\text { Ítem } 3 \text {. Digo malas palabras o cosas que molestan o lastiman a los } \\
\text { otros. }\end{array}$ & & .59 & & .82 & & .70 \\
\hline Ítem 5. Pierdo el control o la calma en casa o en la escuela. & & .59 & & .90 & & .64 \\
\hline $\begin{array}{l}\text { Ítem } 8 \text {. Cuando estoy enojado/a contesto mal a mis padres o maes- } \\
\text { tros. }\end{array}$ & & .63 & & .87 & & .81 \\
\hline$(\rho)$ & .53 & .69 & .95 & .92 & .80 & .77 \\
\hline$\chi_{(19)}^{2}$ & \multicolumn{2}{|c|}{$42.45^{* *}$} & \multicolumn{2}{|c|}{$30.71^{*}$} & \multicolumn{2}{|c|}{$52.80 * *$} \\
\hline CFI & \multicolumn{2}{|c|}{.975} & \multicolumn{2}{|c|}{.995} & \multicolumn{2}{|c|}{.976} \\
\hline NFI & \multicolumn{2}{|c|}{.955} & \multicolumn{2}{|c|}{.987} & \multicolumn{2}{|c|}{.964} \\
\hline NNFI & \multicolumn{2}{|c|}{.963} & \multicolumn{2}{|c|}{.993} & \multicolumn{2}{|c|}{.965} \\
\hline IFI & \multicolumn{2}{|c|}{.975} & \multicolumn{2}{|c|}{.995} & \multicolumn{2}{|c|}{.976} \\
\hline RMSEA & \multicolumn{2}{|c|}{.050} & \multicolumn{2}{|c|}{.056} & \multicolumn{2}{|c|}{.071} \\
\hline
\end{tabular}

Nota. Los ítems de ejemplo corresponden al informante niño. ${ }^{*} p<.05,{ }^{* *} p<.01$. 
Tabla 2

Estadísticos descriptivos, correlaciones entre dominios e inter-informante.

\begin{tabular}{|c|c|c|c|c|c|c|}
\hline & 1 & 2 & 3 & 4 & 5 & 6 \\
\hline 1. Dominio Interpersonal (niño) & - & & & & & \\
\hline 2. Dominio Escolar (niño) & $.40^{* *}$ & - & & & & \\
\hline 3. Dominio Interpersonal (docente) & $.22^{* *}$ & .10 & - & & & \\
\hline 4. Dominio Escolar (docente) & $.14^{*}$ & $.18^{* *}$ & $.51^{* *}$ & - & & \\
\hline 5. Dominio Interpersonal (padre) & $.15^{* *}$ & $.13^{* *}$ & $.20^{* *}$ & $.15^{*}$ & - & \\
\hline 6. Dominio Escolar (padre) & $.09^{*}$ & $.27^{* *}$ & $.20^{* *}$ & $.32^{* *}$ & $.46^{* *}$ & - \\
\hline $\mathrm{M}(\mathrm{DE})$ & $\begin{array}{c}2.22 \\
(0.94)\end{array}$ & $\begin{array}{c}1.75 \\
(0.85)\end{array}$ & $\begin{array}{c}1.55 \\
(0.91)\end{array}$ & $\begin{array}{c}1.33 \\
(0.69)\end{array}$ & $\begin{array}{c}2.01 \\
(1.05)\end{array}$ & $\begin{array}{c}2.11 \\
(1.01)\end{array}$ \\
\hline
\end{tabular}

Nota. $* p<.05 ; * * p<.01$.

casos, e incluso las cargas factoriales estuvieron por encima del mínimo (> .38).

Una vez que se obtuvieron los ítems de cada factor, se calcularon las correlaciones de los dominios inter-informante (niño-padre, $\mathrm{n}=352$; niño-docente, $n=195$; padre-docente, $n=157$ ). Los resultados se presentan en la Tabla 2.

Como puede observarse, las correlaciones entre los dominios escolar e interpersonal son moderadas (niño: $r=.40, p<.01$; docente: $r=$ $.51, p<.01$; padre: $r=.46, p<.01$ ), mientras que las correlaciones entre informantes para el dominio interpersonal (niño-docente: $r=.22, p<.01$; niño-padre: $r=.15, p<.01$; padre-docente: $r=$ $.20, p<.01$ ), y para el dominio escolar (niño-docente: $r=.18, p<.01$; niño-padre: $r=.27, p<$ .01 ; padre-docente: $r=.32, p<.01$ ) fueron bajas.

\section{Objetivo 2: Validez concurrente}

Se evaluó la validez externa (concurrente) mediante correlaciones con las subescalas transgresión a las normas, impulsividad, hiperactividad y déficit atencional de la GOC, y las dimensiones interpersonal y escolar de la EIEDN. Los resultados de dichos análisis, así como los valores estadísticos descriptivos de las variables bajo estudio, se expresan en la Tabla 3.

Puede observarse que las subescalas de la
GOC presentaron correlaciones lineales positivas de moderadas a altas tanto con el dominio escolar como con el dominio interpersonal.

\section{Objetivo 3: Validez convergente}

Para aportar evidencias de validez externa (convergente) en el dominio escolar, se analizaron las relaciones entre la impulsividad escolar (medida a través del informe de padres y docentes) y las habilidades académicas de cálculo y comprensión lectora evaluadas a través de pruebas estandarizadas. Como fue mencionado previamente, debido a la baja fiabilidad del cuestionario de impulsividad respondido por los niños, solo se analizó lo informado por el docente y por los padres. Los resultados se muestran en la tabla 4.

En el dominio escolar, la impulsividad informada por los padres presentó asociaciones inversas bajas con las habilidades de cálculo matemático y comprensión lectora, mientras que la impulsividad informada por el docente presentó una relación inversa moderada solo con el cálculo matemático. Es decir que mientras mayores fueron los puntajes de impulsividad en el dominio escolar, menor fue el desempeño obtenido por los niños en las pruebas de habilidades académicas. 
Tabla 3

Estadísticos descriptivos y correlaciones entre la EIEDN y las dimensiones de la GOC completadas por los docentes.

\begin{tabular}{|c|c|c|c|c|c|c|}
\hline & 1 & 2 & 3 & 4 & 5 & 6 \\
\hline 1. EIEDN-Interpersonal - Docente & - & & & & & \\
\hline 2. EIEDN-Escolar - Docente & $.77 * *$ & - & & & & \\
\hline 3. GOC - Transgresión a las normas & $.65^{* *}$ & $.60 * *$ & - & & & \\
\hline 4. GOC - Impulsividad & $.73 * *$ & $.67 * *$ & $.57 * *$ & - & & \\
\hline 5. GOC - Hiperactividad & $.55 * *$ & $.34 * *$ & $.41 * *$ & $.77 * *$ & - & \\
\hline 6. GOC-Déficit Atencional & $.63 * *$ & $.66^{* *}$ & $.47 * *$ & $.91 * *$ & $.73 * *$ & - \\
\hline $\mathrm{M}(\mathrm{DE})$ & $\begin{array}{c}1.18 \\
(0.80) \\
\end{array}$ & $\begin{array}{c}1.36 \\
(0.97)\end{array}$ & $\begin{array}{c}1.02 \\
(0.05)\end{array}$ & $\begin{array}{c}1.19 \\
(0.42) \\
\end{array}$ & $\begin{array}{c}1.13 \\
(0.38) \\
\end{array}$ & $\begin{array}{c}1.15 \\
(0.40) \\
\end{array}$ \\
\hline
\end{tabular}

Nota. $* p<.05 ; * * p<.01$

Tabla 4

Estadísticos descriptivos y correlaciones entre la EIEDN en su dimensión escolar y pruebas estandarizadas de desempeño académico.

\begin{tabular}{llllc}
\hline & $\mathbf{1}$ & $\mathbf{2}$ & $\mathbf{3}$ & $\mathbf{4}$ \\
\hline 1. EIEDN - docente-escolar & - & & & \\
2. EIEDN - padre-escolar & $.35^{* *}$ & - & & \\
3. Cálculo matemático & $-.38^{* *}$ & $-.23^{*}$ & - & \\
4. Comprensión lectora & -.14 & $-.22^{*}$ & $.20^{*}$ & - \\
\hline $\mathrm{M}(\mathrm{DE})$ & $1.51(0.83)$ & $2.11(0.95)$ & $15.50(3.32)$ & $15.04(4.06)$ \\
\hline
\end{tabular}

Nota. $* p<.05 ; * * p<.01$

\section{Discusión}

Si bien la investigación empírica y teórica en el campo de la impulsividad se ha incrementado notablemente y ha mostrado la importancia de la impulsividad en una variedad de dominios tales como la salud física y mental, el desempeño académico, los hábitos de alimentación y las relaciones interpersonales, los estudios se han focalizado principalmente en rasgos individuales. Por este motivo, la principal contribución del presente estudio fue aportar evidencias de validez para la EIEDN: un instrumento de evaluación de la impulsividad en niños.

En función del Objetivo 1 del estudio (analizar la validez de constructo del modelo bifactorial de la escala), los resultados mostraron un buen ajuste para un modelo de dos factores -escolar e interpersonal-discriminados para cada uno de los informantes (niño, padre y docente). Estos hallazgos concuerdan con los trabajos de Tsukayama et al. (2013) y Mischel et al. (2002), en los que se plantea que los niños - del mismo modo que los adultos- se caracterizan no solo por diferencias individuales, sino también por patrones distintivos y estables de relaciones situación-comportamiento (p. ej., el niño realiza la conducta $\mathrm{A}$ en la situación $\mathrm{B}$, pero realiza la conducta $\mathrm{C}$ en la situación $\mathrm{D})$. En este sentido, se observan relaciones moderadas entre los dominios escolar e interpersonal para cada uno de los informantes. Además, los análisis factoriales apoyan la independencia relativa de los dominios escolar e interpersonal en los diferentes informantes, mostrando que las conductas impulsivas de los niños pueden variar según el contexto en el que se llevan a cabo.

Respecto del grado de acuerdo entre los informantes, se observó que las relaciones para los dominios interpersonal y escolar fueron bajas. Al respecto, De los Reyes et al. (2013) señalan que, en general, es esperable observar relaciones bajas o incluso discrepancias cuando los informantes 
completan medidas paralelas o idénticas. A menudo, los informantes suelen no estar de acuerdo respecto de observaciones comportamentales ( $\mathrm{p}$. ej., De los Reyes et al., 2011). Estas discrepancias pueden representar variaciones en las expresiones de los comportamientos evaluados en los contextos o ambientes, ya que la conducta puede variar según el lugar y, por tanto, según los informantes (por ejemplo, hogar vs. escuela, hogar vs. club). Incluso, la literatura señala que estas discrepancias son el resultado de las diferencias entre las reacciones de los propios informantes ante el comportamiento del niño evaluado (p. ej., Duckworth \& Yeager, 2015). Asimismo, resulta esperable encontrar este patrón de correlaciones cuando no hay psicopatología, porque las conductas varían en función del ambiente, debido al cambio de reglas, pautas y normas de convivencia entre el ámbito escolar y la vida cotidiana en el hogar (De los Reyes \& Kazdin 2005; Dumenci, Achenbach, \& Windle, 2011; Kraemer et al., 2003). Cabe mencionar que estos hallazgos pueden afectar profundamente la forma en que los investigadores interpretan empíricamente sus datos y cómo los profesionales interpretan los resultados de la evaluación en la práctica clínica.

Respecto del Objetivo 2 del estudio (analizar la validez concurrente mediante su asociación con otras medidas), puede observarse que las subescalas transgresión, impulsividad, hiperactividad y déficit de atención de la GOC presentaron asociaciones positivas tanto con la dimensión interpersonal como con la dimensión escolar, según los informes de los docentes. Como fue mencionado, la subescala transgresión a las normas se refiere al incumplimiento de normas sociales apropiadas a la edad, y si bien en sí mismo no es un factor determinante de la impulsividad, es el resultado de un patrón de conductas desajustadas en el aula, debido a que los niños que presentan mayor impulsividad suelen transgredir las normas (en este caso escolares). Por su parte, la subescala de impulsividad de la GOC mostró un patrón de relaciones fuertes con ambas dimensiones de la EIEDN: la escolar y la interpersonal. Cabe mencionar que la impulsividad, especialmente desde la apreciación de la GOC, refiere a un patrón caracterizado por impaciencia, respuestas precipitadas y dificultades para respetar turnos. En tercer lugar, la subescala de hiperactividad se refiere habitualmente a estar inquieto en el asiento del aula, y correr y/o saltar aun en ocasiones en que dicha conducta no es adecuada; y si bien la hiperactividad no es solo motora, sino también intelectual y verbal, este patrón de conductas en el aula suele ser caracterizado como impulsivo. Finalmente, la subescala de déficit de atención indaga sobre si el niño comete errores por descuido con frecuencia, realiza ciertas actividades sin reflexión aparente o presenta dificultades para concluir una tarea. En el ámbito escolar, si la tarea a realizar requiere esfuerzo y concentración, ello provoca rechazo y mala conducta, que a menudo son asociados con la impulsividad. Cabe mencionar que entre las problemáticas que suelen vincularse a la impulsividad (o los fallos en el autocontrol) se encuentran este tipo de conductas externalizantes evaluadas por la GOC, las cuales han recibido particular atención por ser observadas a menudo en el ámbito escolar y presentarse con frecuencia en la infancia (Gomar, Mandil, \& Bunge, 2010; Ison \& Fachinelli, 1993). Estas conductas en general son identificadas como problemáticas y son una causa frecuente de consulta psicológica, aunque no necesariamente constituyen una patología. En conjunto, el patrón de correlaciones obtenido entre las subescalas de la GOC y las medidas de impulsividad escolar e interpersonal contribuyen a robustecer las evidencias de validez del instrumento EIEDN.

Finalmente, respecto del Objetivo 3 del estudio (analizar la validez convergente de la escala 
de impulsividad en el dominio escolar mediante su asociación con variables teóricamente relacionadas), el análisis mostró que mientras mayor fue la impulsividad reportada en el dominio escolar, menor fue el desempeño de los niños en las pruebas de habilidades académicas. Estos resultados concuerdan con los de estudios previos (p. ej., De Ridder et al., 2012; Duckworth, Gendler, \& Gross, 2014; West et al., 2016) que han presentado relaciones bajas pero significativas entre la impulsividad y diferentes pruebas de desempeño académico. Específicamente, el informe de los padres mostró asociaciones con ambas habilidades (cálculo y comprensión lectora), mientras que el informe del docente mostró relaciones solo con la habilidad de cálculo matemático. Esta diferencia en las correlaciones podría indicar que ambos informantes (padres y docentes) evalúan la conducta del niño desde lugares diferentes. En general, los padres describen los comportamientos en un sentido global de la impulsividad, en términos de la conducta que observan fuera de la escuela. A su vez, cabe mencionar que los padres tienen un acceso directo a la conducta del niño en situaciones de lectura o comprensión, más que a situaciones vinculadas con las matemáticas. En este sentido, el docente como informante tiene una mayor especificidad sobre la impulsividad en el ámbito escolar, e incluso puede focalizarse con mayor precisión sobre estos comportamientos, por lo que posiblemente su informe en el dominio escolar resulte más preciso y no global.

En líneas generales, la escala de impulsividad específica de dominio desarrollada por Tsukayama et al. (2013) y traducida al español por Richard's et al. (2017) presenta adecuadas evidencias empíricas de validez. Sin embargo, aun cuando los análisis de validez interna (de constructo) y externa (convergente y concurrente) presentan resultados consistentes, una mención especial debe realizarse respecto de su confiabilidad. El análisis de la confiabilidad de la EIEDN reveló valores aceptables para los docentes y los padres, y un valor adecuado para el dominio social en los niños. No obstante, no fue aceptable para los niños en el dominio escolar. Cabe destacar que resultados similares de baja confiabilidad en instrumentos de autoadministración para niños de edad escolar han sido informados por otros estudios (p. ej., Castrillón-Moreno \& Borrero-Copete, 2005; Ipina, Molina, \& Reyna, 2011). Además, los autores originales de la DSIS-C (Tsukayama et al., 2013) también informan un índice de confiabilidad bajo $(\alpha=.63)$ en ese dominio para el caso de los niños de la misma edad. Al respecto, se ha señalado en la literatura que los niños pequeños pueden sobreestimar la valoración que realizan de sus conductas, porque se encuentran aún en desarrollo las habilidades cognitivas de introspección que les permiten integrar información sobre sus destrezas, y además porque tienen una inclinación a responder de acuerdo a pautas normativas sobre lo que es apropiado hacer o no (Ross, 2006). Desde un enfoque psicométrico, la baja confiabilidad hallada también podría responder a ciertas características específicas de la escala, como la baja cantidad de ítems. Según Loewenthal (2001), existen ocasiones en donde es aceptable un criterio menor al evaluar la confiabilidad de los instrumentos, a saber, cuando se trata de escalas con escaso número de ítems o cuando poseen buenas evidencias de validez. En función de lo anterior, se considera necesario establecer líneas de investigación futuras en relación con la aplicación de medidas como la EIEDN. Asimismo, también es esperable que estudios futuros indaguen sobre la utilidad de esta medida en el ámbito clínico, así como la especificidad y sensibilidad de la escala en general, en su relación con otros síntomas psicopatológicos como la inatención y la hiperactividad, y analicen la confiabilidad de la escala para niños con otros métodos como el análisis test-retest. 
En líneas generales, los resultados del presente estudio posibilitan brindar evidencias de validez de una escala de impulsividad aplicable en Argentina y suponen un avance respecto del uso de una herramienta de evaluación que brinda una mirada integradora de la impulsividad (en el ámbito interpersonal y escolar), y que es accesible y de fácil aplicación. A su vez también brinda la posibilidad de contar con la visión de varias fuentes cercanas al niño, lo cual permite tener una medida general de la impulsividad fortalecida por la evaluación de varios informantes. Si bien futuros estudios resultan necesarios, este estudio constituye un disparador inicial para evaluar la impulsividad de manera general y específica en los ámbitos de la vida cotidiana de los niños en edad escolar.

\section{Referencias}

Abusamra, V., Ferreres, A., Raiter, A., De Beni, R., \& Cornoldi, C. (2010). Test Leer para Comprender TLC. Evaluación de la comprensión de textos. Buenos Aires, Argentina: Paidós.

American Psychological Association. (2010). Ethical principles of psychologists and code of conduct. Washington, DC: American Psychological Association. Recuperado de https://www.apa.org

Andrés, M. L. (2014). Efecto mediador de las estrategias cognitivas de regulación emocional en la relación entre los rasgos de personalidad y la ansiedad, depresión y felicidad en niños de 9 a 12 años de edad (Tesis doctoral). Recuperado de http://rpsico.mdp. edu.ar

Barratt, E. S., Stanford, M. S., Kent, T. A., \& Felthous, A. (1997). Neuropsychological and cognitive psychophysiological substrates of impulsive aggression. Biological Psychiatry, 41(10), 1045-1061. doi: 10.1016/S0006-3223(96)00175-8

Baumeister, R. F., Vohs, K. D., \& Tice, D. M. (2007). The strength model of self-control. Current Direc- tions in Psychological Science, 16(6), 351-355. doi: 10.1111/j.1467-8721.2007.00534.x

Bentler, P. M. (1990). Comparative fit indexes in structural models. Psychological Bulletin, 107(2), 238-246. doi: 10.1037/0033-2909.107.2.238

Bentler, P. M., \& Bonett, D. G. (1980). Significance tests and goodness of fit in the analysis of covariance structures. Psychological Bulletin, 88(3), 588-606. doi: 10.1037/0033-2909.88.3.588

Canet-Juric, L., Galli, J. I., Zaidán, C., \& Vázquez-Echeverría, A. (2019). Autocontrol y desempeño escolar en lengua y matemática. Investigaciones en Psicología, 24(1), 35-42. Recuperado de http:/www.psi.uba.ar/ institucional.php?var=investigaciones/revistas/investigaciones/investigaciones.php

Carretero-Dios, H., \& Pérez, C. (2005). Normas para el desarrollo y revisión de estudios instrumentales. International Journal of Clinical and Health Psychology, 5(3), 521-551. Recuperado de https://www.redalyc. org

Castrillón-Moreno, D., \& Borrero-Copete, P. (2005). Validez estructural y confiabilidad del Inventario de Ansiedad Estado-Rasgo STAIC para la valoración de la ansiedad en niños escolarizados entre 8 y 15 años de la ciudad de Medellín, Colombia. Suma Psicológica, 12(1), 47-60. Recuperado de http://publicaciones. konradlorenz.edu.co/index.php/sumapsi/index

CONICET (2006). Resolución $\mathrm{N}^{\circ} 2857$. Lineamientos para el comportamiento ético en las Ciencias Sociales y Humanidades. Buenos Aires, Argentina. Recuperado de http:/web.conicet.gov.ar/documents/11716/0/ RD+20061211-2857.pdf

De los Reyes, A., \& Kazdin, A. E. (2005). Informant discrepancies in the assessment of childhood psychopathology: A critical review, theoretical framework, and recommendations for further study. Psychological Bulletin, 131(4), 483-509. doi: 10.1037/00332909.131.4.483

De los Reyes, A., Thomas, S. A., Goodman, K. L., \& Kundey, S. M. A. (2013). Principles underlying the use of multiple informants' reports. Annual Review of 
Clinical Psychology, 9(1), 123-149. doi: 10.1146/annurev-clinpsy-050212-185617

De los Reyes, A., Youngstrom, E. A., Swan, A. J., Youngstrom, J. K., Feeny, N. C., \& Findling, R. L. (2011). Informant discrepancies in clinical reports of youths and interviewers' impressions of the reliability of informants. Journal of Child and Adolescent Psychopharmacology, 21(5), 417-424. doi: 10.1089/ cap.2011.0011

De Ridder, D. T. D., Lensvelt-Mulders, G., Finkenauer, C., Stok, F. M., \& Baumeister, R. F. (2012). Taking stock of self-control: A meta-analysis of how trait self-control relates to a wide range of behaviors. Personality and Social Psychology Review, 16(1), 76-99. doi: 10.1177/1088868311418749

Duckworth, A. L., Gendler, T. S., \& Gross, J. J. (2014). Self-control in school-age children. Educational Psychologist, 49(3), 199-217. doi: 10.1080/00461520.2014.926225

Duckworth, A. L., \& Kern, M. L. (2011). A meta-analysis of the convergent validity of self-control measures. Journal of Research in Personality, 45(3), 259-268. doi: 10.1016/j.jrp.2011.02.004

Duckworth, A. L., Kim, B., \& Tsukayama, E.., \& Kim, B. E. (2013). Life stress impairs self-control in early adolescence. Frontiers in Psychology, 3, 608. doi: 10.3389/fpsyg.2012.00608

Duckworth, A. L., Taxer, J. L., Eskreis-Winkler, L., Galla, B. M., \& Gross, J. J. (2019). Self-control and academic achievement. Annual Review of Psychology, 70(1), 373-399. doi: 10.1146/annurev-psych-010418-103230

Duckworth, A. L., \& Yeager, D. S. (2015). Measurement matters: Assessing personal qualities other than cognitive ability for educational purposes. Educational Researcher, 44(4), 237-251. doi: 10.3102/0013189X15584327

Dumenci, L., Achenbach, T. M., \& Windle, M. (2011). Measuring context-specific and cross-contextual components of hierarchical constructs. Journal of Psychopathology and Behavioral Assessment, 33(1),
3-10. doi: 10.1007/s10862-010-9187-4

Eysenck, S. B., \& Eysenck, H. J. (1977). The place of impulsiveness in a dimensional system of personality description. British Journal of Social and Clinical Psychology, 16(1), 57-68. doi: 10.1111/j.20448260.1977.tb01003.x

Few, L. R., Lynam, D. R., \& Miller, J. D. (2015). Impulsivity-related traits and their relation to DSM-5 section II and III personality disorders. Personality Disorders: Theory, Research, and Treatment, 6(3), 261-266. doi: 10.1037/per0000120

George, D., \& Mallery, P. (2003). Using SPSS for Windows step by step: A simple guide and reference. Boston, MA: Allyn \& Bacon.

Gomar, M., Mandil, J., \& Bunge, E. (2010). Manual de terapia cognitiva comportamental con niños y adolescentes. Buenos Aires, Argentina: Polemos.

Guion, K., Mrug, S., \& Windle, M. (2009). Predictive value of informant discrepancies in reports of parenting: Relations to early adolescents' adjustment. Journal of Abnormal Child Psychology, 37(1), 17-30. doi: 10.1007/s10802-008-9253-5

Hair, J. F., Anderson, R. E., Tatham, R. L., \& Black, W. C. (1995). Multivariate data analysis with readings (4a ed). New York: Macmillan.

Hernández-Sampieri, R., Fernández-Collado, C., \& Baptista-Lucio, P. (2014). Metodología de la Investigación (6 $6^{\text {e }}$ d.). México DF: McGraw-Hill Interamericana.

Hu, L., \& Bentler, P. M. (1998). Fit indices in covariance structure modeling: Sensitivity to underparameterized model misspecification. Psychological Methods, 3(4), 424-453. doi: 10.1037/1082-989X.3.4.424

Humphrey, L. L. (1982). Children's and teachers' perspectives on children's self-control: The development of two rating scales. Journal of Consulting and Clinical Psychology, 50(5), 624-633. doi: 10.1037/0022006X.50.5.624

IBM Corp. (2013). IBM SPSS Statistics for Windows (Versión 22.0) [Software de cómputo]. New York: IBM Corp.

Ipina, M. J., Molina, L., \& Reyna, C. (2011). Propiedades 
psicométricas de la Escala MESSY (versión autoinforme) en niños argentinos. Revista de Psicología (PUCP), 29(2), 245-264. Recuperado de http://revistas.pucp.edu.pe/index.php/psicologia/index

Ison, M. S., \& Fachinelli, C. C. (1993). Guía de observación comportamental para niños. Interdisciplinaria, 12(1), 11-21.

Ison, M. \& Soria, E. R. (1997). Baremo de la Guía de Observación Comportamental para Niños. Interdisciplinaria, 14(1-2), 25-46.

Klonsky, E. D., Jane, J. S., Turkheimer, E., \& Oltmanns, T. F. (2002). Gender role and personality disorders. Journal of Personality Disorders, 16(5), 464-476. doi: 10.1521/pedi.16.5.464.22121

Kraemer, H. C., Measelle, J. R., Ablow, J. C., Essex, M. J., Boyce, W. T., \& Kupfer, D. J. (2003). A new approach to integrating data from multiple informants in psychiatric assessment and research: Mixing and matching contexts and perspectives. American Journal of Psychiatry, 160(9), 1566-1577. doi: 10.1176/appi. ajp.160.9.1566

Loewenthal, K. M. (2001). An introduction to psychological tests and scales ( $2^{\mathrm{a}}$ ed.). East Sussex: Psychology Press.

Mischel, W., Shoda, Y., \& Mendoza-Denton, R. (2002). Situation-behavior profiles as a locus of consistency in personality. Current Directions in Psychological Science, 11(2), 50-54. doi: 10.1111/1467-8721.00166

Morelato, G., \& Ison, M. S. (2002). Autoconcepto real e ideal en niños: Estudio comparativo. Revista Irice, 16, 35-48. Recuperado de http://www.irice-conicet. gov.ar:8080/portal/pda/9a969dbf-e2ee-4704-8340d 18213 d3eebb/tool/22306f5f-abf1-432a-aeab$3879 \mathrm{e} 245 \mathrm{a} 8 \mathrm{cc}$

Musso, M. (2009). Evaluación de funciones ejecutivas en niños: Análisis y adaptación de pruebas en un contexto escolar. Revista Iberoamericana de Diagnóstico y Evaluación-e Avaliação Psicológica, 27(1), 157-178. Recuperado de https://www.aidep.org/es/ ridep

Nigg, J. T. (2017). Annual Research Review: On the rela- tions among self-regulation, self-control, executive functioning, effortful control, cognitive control, impulsivity, risk-taking, and inhibition for developmental psychopathology. The Journal of Child Psychology and Psychiatry, 58(4), 361-383. doi: 10.1111/jcpp. 12675

Nunnally, J. C. (1978). Psychometric theory (2a ed.). New York, NY: McGraw-Hill.

Patton, J. H., Stanford, M. S., \& Barratt, E. S. (1995). Factor structure of the Barratt impulsiveness scale. Journal of Clinical Psychology, 51(6), 768-774. doi: 10.1002/1097-4679(199511)51:6<768::aid-jclp2270510607>3.0.co;2-1

Richard's, M. M., Introzzi, I., Zamora, E., Vernucci, S., Stelzer, F., \& Andrés, M. L. (2017). Evidencias de validez convergente del paradigma Stop-Signal para la medición de la inhibición comportamental en niños. Revista Argentina de Neuropsicología, 30, 50-65. Recuperado de https://www.revneuropsi.com.ar

Ross, J. A. (2006). The reliability, validity, and utility of self-assessment. Practical Assessment, Research and Evaluation, 11(10), 1-13. Recuperado de https:// scholarworks.umass.edu/pare

Scientific Software International (2006). LISREL (8). [Software de cómputo]. Recuperado de http://www.ssicentral.com

Sireci, S. G. (2003). Validity: Content. En R. Fernández-Ballesteros (Ed.), Encyclopedia of Psychological Assessment. (Vol. 2, pp. 1075-1077). London, UK: Sage.

Snelbaker, A. J., Wilkinson, G. S., Robertson, G. J., \& Glutting, J. J. (2001). Wide Range Achievement Test 3 (WRAT3). En W. I. Dorfman \& M. Hersen (Eds.), Understanding psychological assessment (pp. 259274). New York: Springer US. doi: 10.1007/978-14615-1185-4_13

Squillace, M., \& Picón-Janeiro, J. (2017). Impulsividad, un constructo multifacético: Validación del CUBI. Revista Evaluar, 17(1), 1-17. doi: 10.35670/1667-4545. v17.n1.17070

Squillace, M., Picón-Janeiro, J., \& Schmidt, V. (2011). 
El concepto de impulsividad y su ubicación en las teorías psicobiológicas de la personalidad. Revista Neuropsicología Latinoamericana, 3(1), 8-18. doi: 10.5579/rnl.2011.0057

Tabachnick, B. G., \& Fidell, L. S. (2001). Using multivariate statistics. Boston, MA: Allyn \& Bacon.

Tsukayama, E., Duckworth, A. L., \& Kim, B. (2013). Domain specific impulsivity in school-age children. Developmental Science, 16(6), 879-893. doi: 10.1111/desc. 12067

Vernucci, S., Canet-Juric, L., Andrés, M. L., \& Burin, D. I. (2017). Comprensión lectora y cálculo matemático: El rol de la memoria de trabajo en niños de edad escolar. Psykhe, 26(2), 1-13. doi: 10.7764/ psykhe.26.2.1047

West, M. R., Kraft, M. A., Finn, A. S., Martin, R. E., Duckworth, A. L., Gabrieli, C. F. O., \& Gabrieli, J. D. E. (2016). Promise and paradox: Measuring students' non-cognitive skills and the impact of schooling. Educational Evaluation and Policy Analysis, 38(1), 148-170. doi: 10.3102/0162373715597298

Whiteside, S. P., \& Lynam, D. R. (2001). The Five Factor Model and impulsivity: Using a structural model of personality to understand impulsivity. Personality and Individual Differences, 30(4), 669-689. doi: 10.1016/S0191-8869(00)00064-7

Willcutt, E. G., Nigg, J. T., Pennington, B. F., Solanto, M. V., Rohde, L. A., Tannock, R. ... \& Lahey, B. B. (2012). Validity of DSM-IV attention deficit/hyperactivity disorder symptom dimensions and subtypes. Journal of Abnormal Psychology, 121(4), 991-1010. doi: $10.1037 / \mathrm{a} 0027347$

Wilkinson, G. S. (1993). Wide Range Achievement Test 3. Wilmington, DE: Wide Range.

Wilkowski, B. M., \& Robinson, M. D. (2016). Cognitive control processes underlying individual differences in self-control. En E. R. Hirt, J. J. Clarkson \& L. Jia (Eds.), Self-regulation and ego control (pp. 301-324). Academic Press. doi: 10.1016/B978-0-12801850-7.00015-9

World Medical Association. (2013). Declaration of Helsin- ki - Ethical principles for medical research involving human subjects. Recuperado de https://www.wma. net

Zapolski, T. C. B., Stairs, A. M., Settles, R. F., Combs, J. L., \& Smith, G. T. (2010). The measurement of dispositions to rash action in children. Assessment, 17(1), 116-125. doi: 10.1177/1073191109351372

Zuckerman, M. (2007). The sensation seeking scale V (SSS-V): Still reliable and valid. Personality and Individual Differences, 43(5), 1303-1305. doi: 10.1016/j.paid.2007.03.021

Zuckerman, M., Eysenck, S. B., \& Eysenck, H. J. (1978). Sensation seeking in England and America: cross-cultural, age, and sex comparisons. Journal of Consulting and Clinical Psychology, 46(1), 139149. doi: 10.1037/0022-006X.46.1.139 